\title{
ENDOMORPHISM RINGS OF OPERATOR LOOPS
}

\author{
BY \\ REINHOLD BAER
}

If $L$ is an operator loop, then it is impossible to define the addition of any two admissible endomorphisms of $L$, unless $L$ has a somewhat special structure, for example is a commutative group. To cope with this situation, $\mathrm{H}$. Fitting [1]( $\left.{ }^{1}\right)$ has introduced a generalized type of rings which comprises the systems of admissible and normal endomorphisms of operator groups. If one wants, however, to retain the ordinary ring nature of the system of admissible endomorphisms without sacrificing at the same time the generality of the structure of the operator loop $L$, then one is naturally forced to restrict the class of endomorphisms under consideration. Thus we propose to consider in the present investigation the system $\theta$ of all those admissible endomorphisms of $L$ which map $L$ into some preassigned commutative subgroup $A$ of $L$. Then $\theta$ is an ordinary ring which is intimately connected with the situation of $A$ in $L$. The greater generality in the structure of the pair $A, L$ makes for greater variety in the nature of the ring $\theta$. For this ring may or may not have an identity; it may have a right identity, but no left identity; it may or may not be equal to its radical, and so on.

Assuming now that every endomorphism in $\theta$ splits $L$, in the sense of Baer [4], we may show that $\theta$ enjoys many of the properties, usually only proved on the basis of chain conditions for the ideals in $\theta$. We mention only a few of them. Right quasi regularity, in the sense of Perlis [1], implies left quasi regularity; the radical of $\theta$, in the sense of Jacobson [2], is the compositum of all the right ideals without idempotents, not 0 ; primarity criteria may be extended also.

1. Definition of the ring $\theta$. We consider a loop $L$ which admits as operators the elements in a certain set $M$. Thus there is defined for any elements $x$ and $y$ in $L$ their sum $x+y$, meeting the customary requirements, and there exists for every element $m$ in $M$ and every $x$ in $L$ their product $m x$, meeting the requirement $m(x+y)=m x+m y$. As usual we term the subloop $S$ of $L$ admissible or an $M$-subloop of $L$ if $M S \leqq S$. For further remarks concerning the elements of operator loop theory see, for example, Baer [3].

An $M$-endomorphism $\eta$ of $L$ is a single-valued $L$-to- $L$ mapping satisfying

$$
(x+y) \eta=x \eta+y \eta \text { and }(m x) \eta=m(x \eta)
$$

for $x, y$ in $L$ and $m$ in $M$. Every $M$-endomorphism $\eta$ of $L$ maps $L$ upon an

Presented to the Society, November 30, 1946; received by the editors July 27, 1946.

(1) Numbers in brackets refer to the bibliography at the end of the paper. 
$M$-subloop $L \eta$ of $L$ and, more generally, it maps $M$-subloops upon $M$-subloops.

If $\eta$ and $\kappa$ are $M$-endomorphisms of $L$, then we define as usual their product $\eta \kappa$ by the formula

$$
(x \eta) \kappa=x(\eta \kappa)
$$

for $x$ in $L$, and we define their sum $\eta+\kappa$ by the rule:

$$
x \eta+x \kappa=x(\eta+\kappa)
$$

for $x$ in $L$. The product of $M$-endomorphisms is again an $M$-endomorphism; this multiplication is associative, but the sum of $M$-endomorphisms need not be an $M$-endomorphism; see in this context Fitting [1]. To obtain rings of endomorphisms in the customary sense we have to consider suitably selected subsets of the set of all the $M$-endomorphisms of $L$; this will be done as follows:

Let $A$ be an $M$-subloop of $L$ which is an abelian group (with respect to the addition of elements) or as we shall say shortly: $A$ is an admissible abelian subgroup of $L$. Denote by $\theta=\theta(A)$ the system of all the $M$-endomorphisms of $L$ which map $L$ into $A$. It is readily seen that they form an ordinary ring with respect to addition and multiplication as defined above. These rings $\theta=\theta(A)$ will be the object of the following investigation.

Of particular interest will be the idempotent elements in $\theta$. They are admissible endomorphisms, mapping $L$ into $A$, and satisfying $\epsilon^{2}=\epsilon$. The image of $L$ under such an idempotent $\epsilon$ shall be termed a retract of $L$. They are retracts of $L$ in the customary sense of the word which are at the same time admissible subgroups of $A$.

If $R$ is a retract of $L$, then there exists an idempotent endomorphism $\rho$ in $\theta$, mapping $L$ upon $L \rho=R$. If $K$ is the kernel of $\rho$, then $K$ is a normal subloop of $L ; K$ is admissible and we have:

$$
K \cap R=0, \quad K+R=L .
$$

But as $R$ need not be normal in $L$, this decomposition need not be a direct one.

We denote by $K(\theta)$ the cross cut of the kernels of all the endomorphisms in $\theta$. It is clear that $K(\theta)$ is a normal and admissible subloop of $L$. Since $L \eta \leqq A$ is an ordinary abelian operator group for every $\eta$ in $\theta$, one verifies that $L / K(\theta)$ is an abelian $M$-group too. For if $K(\eta)$ is the kernel of the endomorphism $\eta$ in $\theta$, then $L / K(\eta)$ is an abelian $M$-group so that every $K(\eta)$, and hence $K(\theta)$, contains the commutator-associator subloop of $L$.

By $L \theta$ we shall denote the subgroup of $A$ which is generated by all the elements $x \eta$ for $x$ in $L$ and $\eta$ in $\theta$. It is clear that $L \theta$ is an admissible subgroup of $L$.

Sometimes it will be possible to obtain sharper results by the following special choice of the distinguished abelian subgroup $A$. We say that the ele- 
ment $z$ in $L$ is a center element, if

$$
z+x=x+z
$$

for every $x$ in $L$, and

$$
z+(x+y)=(z+x)+y=x+(z+y)
$$

for $x$ and $y$ in $L$. The center elements of $L$ are readily seen to form an abelian subgroup of the loop $L$, but this subgroup need not be admissible. However, the join of all the admissible subgroups of the group of all the center elements is an $M$-subgroup of $L$ which we term, following Korínek [1], the $M$-center $Z=Z(L)$ of $L$. We note that $Z$ and every $M$-subgroup of $Z$ is an abelian and normal subgroup of $L$.

2. Existence of identity. If $A$ is any admissible and abelian subgroup of the $M$-loop $L$, then the identity automorphism of $L$ will belong to $\theta(A)$ if, and only if, $A=L$. However, even though $A \neq L$, it is possible that the ring $\theta$ contains an identity element. The present section is devoted to a discussion of this question.

Let us recall first that the element $\epsilon$ in $\theta$ is termed a right (left) identity element, if $\tau \epsilon=\tau(\epsilon \tau=\tau)$ for every $\tau$ in $\theta$; and if $\epsilon$ is both a right and a left identity, then we term $\epsilon$ the identity of $\theta$. It is well known that there exists at most one identity in $\theta$. Note that right and left identity elements are idempotents which will be different from 0 whenever $\theta \neq 0$.

THEOREM 1. There exists a right identity element in $\theta(A)$ if, and only if, $L \theta$ is a retract of $L$.

Proof. If $\epsilon$ is a right identity element of $\theta$, then $L \epsilon \leqq L \theta$, since $\epsilon$ is in $\theta$. But $L \theta=L \theta \epsilon \leqq L \epsilon$. Hence $L \theta=L \epsilon$, showing that $L \theta$ is a retract if $\epsilon$ is a right identity element in $\theta$. If conversely $L \theta$ is a retract of $L$, then there exists an idempotent $\epsilon$ such that $L \theta=L \epsilon$; $\epsilon$ is in $\theta$, since $L \theta \leqq A$. If $\tau$ is in $\theta$ and $x$ in $L$, then $x \tau$ is in $L \theta=L \epsilon$ so that $x \tau \epsilon=x \tau$ or $\tau \epsilon=\tau$, proving that $\tau$ is a right unit.

THEOREM 2. There exists a left identity element in $\theta(A)$ if, and only if, $K(\theta)$ is the kernel of an idempotent endomorphism in $\theta$.

Proof. If $\epsilon$ is a left identity element of $\theta$, then $\epsilon$ is an idempotent in $\theta$ whose kernel $K(\epsilon)$ clearly satisfies $K(\theta) \leqq K(\epsilon)$. If $\eta$ is in $\theta$, and if $x$ is an element in the kernel of $\epsilon$, then we have $x \eta=x \epsilon \eta=0$ so that $x$ belongs to the kernel of $\eta$ too showing $K(\theta)=K(\epsilon)$. If, conversely, $\epsilon$ is an idempotent in $\theta$ whose kernel $K(\epsilon)$ is equal to $K(\theta)$, and if $\eta$ is in $\theta$, then $\eta-\epsilon \eta$ is a well determined element in $\theta$. If $x$ is an element in $L$, then there exists one and only one element $y$ in $L$ such that $x=x \epsilon+y$. Applying $\epsilon$ to this equation we find that $x \epsilon=x \epsilon^{2}+y \epsilon=x \epsilon+y \epsilon$ or $y \epsilon=0$ so that $y$ is in the kernel $K(\epsilon)=K(\theta)$. Hence $y \eta=0$ for every $\eta$ in $\theta$. Consequently $x(\eta-\epsilon \eta)=(x \epsilon+y)(\eta-\epsilon \eta)=(x \epsilon)(\eta-\epsilon \eta)$ $=0$, proving that $\eta-\epsilon \eta=0$ and that $\epsilon$ is left identity of $\theta$. 
CoRollary 1. There exists an identity element of $\theta$ if, and only if, $L \theta$ is a retract of $L$ and $K(\theta)$ is the kernel of an idempotent in $\theta$.

Proof. The necessity of these conditions is a consequence of Theorems 1 and 2 , since identity elements are both right and left identities. If conversely these conditions are satisfied, then we infer from Theorem 1 the existence of a right identity element $\epsilon$ and from Theorem 2 the existence of a left identity element $\boldsymbol{\epsilon}^{\prime}$ in $\theta$. But then $\boldsymbol{\epsilon}=\boldsymbol{\epsilon}^{\prime} \boldsymbol{\epsilon}=\boldsymbol{\epsilon}^{\prime}$, proving that this element is the identity element of $\theta$.

Corollary 2. Assume that $A=Z(L)$ is the $M$-center of $L$ and that $\theta=\theta(Z(L))$ is the ring of all the "center endomorphisms" of $L$.

(a) There exists a right identity element in $\theta$ if, and only if, $L \theta$ is a direct summand of $L$.

(b) There exists a left identity element in $\theta$ if, and only if, $K(\theta)$ is a direct summand of $L$.

(c) There exists an identity element in $\theta$ if, and only if, $L$ is the direct sum of $L \theta$ and $K(\theta)$.

Proof. (a) is an immediate consequence of the fact, pointed out before, that retracts which are part of the center are direct summands. If $K(\theta)$ is a direct summand of $L$, then there exists an admissible subloop $S$ of $L$ such that $L$ is the direct sum of $S$ and $K(\theta)$. We noted in $\S 1$ that $L / K(\theta)$ is an abelian $M$-group. Hence $S$ is an abelian group, and now it is not difficult to verify that $S \leqq Z(L)$. If $\epsilon$ is the idempotent endomorphism of $L$ which leaves invariant every element in $S$ and which maps onto 0 every element in $K(\theta)$, then $\epsilon$ belongs to $\theta$ and has $K(\theta)$ for its kernel. Thus $K(\theta)$ is the kernel of an idempotent in $\theta$, and the existence of a left identity element in $\theta$ may be inferred from Theorem 2-as a matter of fact it is easy to see that $\epsilon$ is a left identity of $\theta$. If conversely there exists a left identity element in $\theta$, then we infer from Theorem 2 that $K(\theta)$ is the kernel of an idempotent $\epsilon$ in $\theta$. But the retract $L \epsilon$ of $L$ is now part of the $M$-center $Z(L)$ of $L$ so that $L$ is the direct sum of $K(\theta)$ and $L \epsilon$, completing the proof of (b).

If $\epsilon$ is the identity element of $\theta$, then we show, as in Theorem 1 , that $L \theta=L \epsilon$; we show, as in Theorem 2 , that $K(\theta)$ is the kernel of $\epsilon$. Since $\epsilon$ is idempotent, and since $L \theta$ is part of the $M$-center $Z(L)$ of $L$, it follows that $L$ is the direct sum of $L \theta$ and $K(\theta)$. If conversely $L$ is the direct sum of $L \theta$ and $K(\theta)$, then we infer from (a) the existence of a right identity element $\epsilon$, and from (b) the existence of a left identity element $\epsilon^{\prime}$. But then $\epsilon=\epsilon^{\prime}$ is the desired identity element of $\theta$.

3. Irreducibility. The admissible subloop $S$ of the $M$-loop $L$ is irreducible whenever 0 and $S$ are its only admissible subloops.

THEOREM. If $L \theta$ is irreducible, and if $\eta$ is an endomorphism in $\theta(A)$ such 
that $\eta^{2} \neq 0$, then there exists a right identity element $\epsilon$ in $\theta$ such that $\epsilon \eta=\eta$ and $\epsilon \theta$ is a (not necessarily commutative) subfield of $\theta$.

Proof. Since $J=L \theta$ is irreducible, it follows that the cross cut of $J$ and of the kernel $K(\eta)$ of $\eta$ is either $J$ or 0 . In the first case $J \leqq K(\eta)$ so that $L \eta^{2} \leqq J \eta \leqq K(\eta) \eta=0$ whch would imply $\eta^{2}=0$, contrary to our hypothesis. Hence 0 is the cross cut of $J$ and $K(\eta)$.

Since $J \eta \leqq L \theta=J$, it follows from the irreducibility of $J$ that $J=J \eta$ or $J \eta=0$. The latter is impossible, since $J \neq 0$, though its intersection with $K(\eta)$ is 0 . Hence $J=J \eta$.

If $x$ is an element in $L$, then $x \eta$ is in $J=J \eta$. Consequently there exists an element $x^{\prime}$ in $J$ such that $x \eta=x^{\prime} \eta$. There exists one and only one element $y$ in $L$ such that $x=x^{\prime}+y$, and it is clear that $y \eta=0$ so that $y$ is in $K(\eta)$. Thus $L=J+K(\eta)$; now it is readily seen that there exists an idempotent endomorphism $\epsilon$ in $\theta$ which leaves invariant every element in $J$ and which maps $K(\eta)$ upon 0 . As in $\S 2$, Theorem 1, it is shown that $\epsilon$ is a right identity element of $\theta$. From $L=J+K(\eta)$ and $L \eta=J=J \eta$ one deduces $\epsilon \eta=\eta$, and by the customary arguments (see, for example, Jacobson $[1$, p. 57, Theorem 4]) one verifies that $\epsilon \theta$ is a field.

That $\theta$ need not be a field is a consequence of the fact that there may exist, under the present circumstances, several right identity elements of $\theta$. If, however, there should exist a left identity element in $\theta$, then there would exist one and only one identity element; the previous arguments would show that $\theta$ is a field. (Cf. Jacobson $[1$, p. 57 , Theorem 4].)

4. Nil endomorphisms. If $\eta$ is any admissible endomorphism of the $M$-loop $L$, then we denote by $R(\eta)$ the set of all the elements $x$ in $L$ with the following property:

There exists an integer $n=n(x)$ such that $x \eta^{n}=0$.

It is easily seen that $R(\eta)$ is a normal and admissible subloop of $L$, since it is just the join of the kernels of the endomorphisms $\eta^{n}$. We shall call this subloop $R(\eta)$ the radical of $\eta$. For elementary properties of the radical of an endomorphism see Baer [4].

The admissible endomorphism $\eta$ of the $M$-loop $L$ shall be termed $a$ nil endomorphism whenever $L=R(\eta)$. If $\eta$ is a nil endomorphism, then every element in $L$ is mapped upon 0 by "almost every" power of $\eta$. See Kaplansky [1] for topological generalizations.

Assume now that $A$ is an admissible abelian subgroup of the $M$-loop $L$ and that the nil endomorphism $\eta$ is in $\theta(A)$. If $n_{i}$ is, for every positive $i$, an integer, and if $x$ is an element in $L$, then $x\left(n_{i} \eta^{i}\right)=0$ for every $i$ with a finite number of exceptions; thus $x \sum_{i=1}^{\infty} n_{i} \eta^{i}$ is a well determined element $x \sigma$ in $A$. It is easy to verify that $\sigma$ is an admissible endomorphism in $\theta$; we shall, therefore, be able to use the notation $\sigma=\sum_{i=1}^{\infty} n_{i} \eta^{i}$. One may prove the fact of which no use will be made in the future that the infinite power series in $\eta$ with in- 
tegral coefficients form a commutative ring which is a homomorphic image of the ring of all the formal power series in one variable with integral coefficients and without absolute term.

THEOREM. Suppose that $\eta$ is a nil endomorphism in $\theta$.

(a) The intersection of the right ideals $\eta^{n} \theta$ for $0<n$ is 0 .

(b) The power series $\sum_{i=1}^{\infty} c_{i} \eta^{i}$ with integral coefficients is uniquely determined as the solution of the congruences:

$$
\sigma \equiv \sum_{i=1}^{n} c_{i} \eta^{i} \operatorname{modulo} \eta^{n+1} \theta
$$

Proof. Suppose that the endomorphism $\kappa$ is in the intersection of all the right ideals $\eta^{n} \theta$. Then there exists to every positive integer $n$ an endomorphism $\kappa_{n}$ in $\theta$ such that $\kappa=\eta^{n} \kappa_{n}$. If $x$ is an element in $L$, then there exists an integer $j=j(x)$ such that $x \eta^{j}=0$, since $\eta$ is a nil endomorphism. Hence $x \kappa=x \eta^{j} \kappa_{j}=0$, proving (a). The contention (b) is readily deduced from (a) and the construction of the power series in the nil endomorphism $\eta$.

Following Perlis [1] we term the element $\rho$ in the ring $\theta$ quasi regular whenever there exists an element $\sigma$ in $\theta$ such that

$$
\rho+\sigma+\rho \sigma=0 \text { and } \rho \sigma=\sigma \rho .
$$

The element $\sigma$ is uniquely determined by $\rho$ and these equations; $\sigma$ may therefore be called the quasi inverse of $\rho$; see Baer [2] and Jacobson [2].

COROLlaRY 1. If $\eta$ is a nil endomorphism in $\theta$, then $\eta$ is quasi regular and its quasi inverse is $\sum_{i=1}^{\infty}(-1)^{i} \eta^{i}$.

This is an almost immediate inference from the preceding discussion. It is easy to construct examples showing that the converse of this statement is not true.

Following Jacobson [2], we define the radical $\mathbf{P}$ of the ring $\theta$ as the join of all the right ideals in $\theta$ which consist of quasi regular elements only. The radical $\mathbf{P}$ of $\theta$ is known to be a two-sided ideal in $\theta$ such that $\theta / \mathbf{P}$ is a semisimple ring (has radical 0 ).

COROLlaRY 2. If every endomorphism in the right ideal in $\theta$ which is generated by $\eta$ is a nil endomorphism, then $\eta$ belongs to the radical $\mathrm{P}$ of $\theta$.

This is an immediate consequence of Corollary 1. Again we cannot claim the validity of the converse of Corollary 2 .

5. Splitting endomorphisms. The admissible subloop $C$ of the $M$-loop $L$ shall be called a complement of the admissible endomorphism $\eta$ of $L$ whenever

(i) $C=C \eta$,

(ii) $C \cap R(\eta)=0$,

(iii) $C+R(\eta)=L$. 
The conditions (ii) and (iii) are equivalent to saying that there exists an idempotent admissible endomorphism with kernel $R(\eta)$ which maps $L$ upon $C$. Whenever there exists a complement of an endomorphism $\eta$, then we shall say that $\eta$ splits $L$ or is a splitting endomorphism; see Baer [4] for this and related concepts.

If $A$ is an admissible abelian subgroup of the $M$-loop $L$, and if $C$ is a complement of the endomorphism $\eta$ in $\theta(A)$, then it follows from condition (i) that $C$ is part of $A$ so that the admissible idempotent endomorphism of kernel $R(\eta)$ and image $C$ belongs to $\theta(A)$ too.

THEOREM. The endomorphism $\eta$ in $\theta(A)$ splits the $M$-loop $L$ if, and only if, there exist endomorphisms $\epsilon$ and $\kappa$ in $\theta$ with the following properties:

$$
\eta \kappa=\kappa \eta=\epsilon, \quad \kappa \epsilon=\epsilon \kappa=\kappa, \quad L=R(\eta-\epsilon \eta) .
$$

Proof. Assume first the existence of a complement $C$ of the endomorphism $\eta$ in $\theta$. Then there exists an idempotent endomorphism $\epsilon$ in $\theta$ whose kernel is $R(\eta)$ and which maps $L$ upon $C$. From condition (i) we infer that $\eta$ induces a proper automorphism $\tau$ in its complement $C$. Consequently there exists one and only one endomorphism $\kappa$ of $L$ which induces $\tau^{-1}$ in $C$ and which maps $R(\eta)$ upon 0 (see conditions (ii) and (iii)!). One verifies readily that $\kappa$ is in $\theta$ and that $\eta \epsilon=\epsilon \eta, \eta \kappa=\kappa \eta=\epsilon, \kappa \epsilon=\epsilon \kappa=\kappa$. If $x$ is an element in $C$, then $x(\eta-\epsilon \eta)$ $=0$; if $x$ is an element in $R(\eta)$, then $x \epsilon=0$ and consequently $x(\eta-\epsilon \eta)^{i}=x \eta^{i}$, proving that $L=R(\eta-\epsilon \eta)$. Thus our conditions have been shown to be necessary.

Assume conversely that there exist endomorphisms $\epsilon$ and $\kappa$ in $\theta$ such that $\eta \kappa=\kappa \eta=\epsilon, \kappa \epsilon=\epsilon \kappa=\kappa, L=R(\eta-\epsilon \eta)$. Then

$$
\epsilon^{2}=(\eta \kappa) \epsilon=\eta(\kappa \epsilon)=\eta \kappa=\epsilon,
$$

proving that $\epsilon$ is an idempotent. Furthermore $\eta \epsilon=\eta(\eta \kappa)=\kappa \eta^{2}=\epsilon \eta$. If $x$ is in $R(\eta)$, then there exists a positive integer. $n$ such that $x \eta^{n}=0$. Hence

$$
x \epsilon=x \epsilon^{n}=x(\eta \kappa)^{n}=x \eta^{n} \kappa^{n}=0 .
$$

If conversely $y$ belongs to the kernel of $\epsilon$, then $y(\eta-\epsilon \eta)^{i}=y \eta^{i}$ for every positive $i$. But $L=R(\eta-\epsilon \eta)$ implies the existence of a positive integer $j$ such that $0=y(\eta-\epsilon \eta)^{i}=y \eta^{j}$; thus we have shown that $R(\eta)$ is exactly the kernel of the idempotent $\epsilon$. Let $C=L \epsilon$. Then $C$ is an admissible subgroup of $A$, since $\epsilon$ is in $\theta$. Since $\epsilon$ is an idempotent whose kernel is $R(\eta)$, it follows that $L=C+R(\eta)$ and $0=C \cap R(\eta)$. Furthermore we have

$$
C \eta=L \epsilon \eta=L \eta \epsilon \leqq L \epsilon=C \quad \text { and } \quad C_{\kappa}=L \epsilon \kappa=L \kappa \epsilon \leqq L \epsilon=C
$$

so that $\eta$ and $\kappa$ induce admissible endomorphisms in $C$. But $\eta \kappa=\kappa \eta=\epsilon$ and $\epsilon$ effects the identity in $C$. Hence $\eta$ and $\kappa$ induce reciprocal automorphisms in $C$, proving in particular that $C=C \eta$. Thus we have shown that $C$ is a complement of $\eta$, completing the proof. 
It may be worth noting that the endomorphism $\epsilon$ constructed is always idempotent and satisfies $\eta \epsilon=\epsilon \eta$.

Appendix on the endomorphism induced by $\eta$ in $\theta$. The ring $\theta$ is an abelian group with respect to addition; it may be turned into an operator group by considering right multiplication by elements in $\theta$. If $\eta$ is an element in $\theta$, then left multiplication by $\eta$ effects an endomorphism of this operator group.

Suppose now that $\eta$ is a splitting endomorphism of $L$ into $A$. Then we infer from the preceding considerations the existence of an idempotent $\epsilon$ in $\theta$ and of an endomorphism $\kappa$ in $\theta$ such that

$$
\epsilon \eta=\eta \epsilon, \quad \eta \kappa=\kappa \eta=\epsilon, \quad \kappa \epsilon=\epsilon \kappa=\kappa, \quad L=R(\eta-\epsilon \eta) ;
$$

from the last identity and the theorem of $\$ 4$ we deduce that the cross cut of the ideals $(\eta-\epsilon \eta)^{i} \theta$ is 0 .

Since $\epsilon$ is an idempotent in $\theta$, it follows that $\theta$ is the direct sum of $\epsilon \theta$ and of the right ideal $\Xi$, consisting of the elements $\tau-\epsilon \tau$ for $\tau$ in $\theta$, and we ask ourselves what left multiplication by $\eta$ does to these right ideals.

(1) $\eta$ effects an automorphism in $\epsilon \theta$.

Proof. We note first that

$$
\eta(\epsilon \theta)=\epsilon \eta \theta \leqq \epsilon \theta=\eta \kappa \epsilon \theta=\eta \epsilon \kappa \theta \leqq \eta(\epsilon \theta) \text { or } \epsilon \theta=\eta(\epsilon \theta) \text {; }
$$

secondly we infer from $0=\eta \epsilon \tau$ for $\tau$ in $\theta$ that $0=\kappa \eta \epsilon \tau=\epsilon^{2} \tau=\epsilon \tau$, completing the proof.

(2) 0 is the intersection of the right ideals $\eta^{i} \Xi$.

Proof. Suppose that $\tau$ is in $\theta$ and that $i$ is a positive integer. Then

$$
\begin{aligned}
(\eta-\epsilon \eta)^{i}(\tau-\epsilon \tau) & =(\eta-\epsilon \eta)^{i-1}(\eta(\tau-\epsilon \tau)-\eta \epsilon(\tau-\epsilon \tau)) \\
& =(\eta-\epsilon \eta)^{i-1} \eta(\tau-\epsilon \tau)=\eta(\eta-\epsilon \eta)^{i-1}(\tau-\epsilon \tau)=\cdots \\
& =\eta^{i}(\tau-\epsilon \tau)
\end{aligned}
$$

so that $\eta^{i} \boldsymbol{\Xi}=(\eta-\epsilon \eta)^{i} \boldsymbol{\Xi} \leqq(\eta-\epsilon \eta)^{i} \theta$; we remarked before that the cross cut of the latter ideals is 0 , proving (2).

(1) and (2) together show that $\theta=\epsilon \theta \oplus \Xi$ is a decomposition of $\theta$ which, with respect to the endomorphism $\eta$ of $\theta$, constitutes a kind of splitting, different from the one considered thus far.

6. Rings of splitting endomorphisms. From now on we shall impose upon the admissible abelian subgroup $A$ of the $M$-loop $L$ the following hypothesis (without actually always restating it):

(F) Every endomorphism in the ring $\theta(A)$ splits $L$.

This condition is a weak form of chain condition, since, for instance, Fitting's lemma asserts that every admissible endomorphism of an operator group with double chain condition splits; see Fitting [2] and for more general splitting theorems see Baer [4].

THEOREM 1. If the admissible retract $R$ of the $M$-loop $L$ is part of $A$, and if $\rho$ 
is an admissible isomorphism of $R$ into itself, then $R=R \rho$.

Proof. There exists an idempotent and admissible endomorphism $\epsilon$ of the $M$-loop $L$ such that $L \epsilon=R$. Consequently $\epsilon \rho$ is a well determined admissible endomorphism of $L$ which maps $L$ upon $L \epsilon \rho=R \rho \leqq R \leqq A$. Hence $\epsilon \rho$ is in $\theta(A)$; the existence of a complement $C$ of $\epsilon \rho$ follows from $(\mathrm{F})$. It is readily seen that $R(\epsilon \rho)$ is the kernel of $\epsilon$, and we have furthermore

$$
C=C \epsilon \rho \leqq R \rho \leqq R .
$$

But $L=R+R(\epsilon \rho)=C+R(\epsilon \rho)$ whereas $R \cap R(\epsilon \rho)=0$. Hence $R=C$, and thus $R=R \rho$ is a consequence of a previous inequality.

THEOREM 2. The endomorphism $\eta$ in $\theta(A)$ is a nil endomorphism if, and only if, 0 is the intersection of the right ideals $\eta^{n} \theta$ for $0<n$.

Proof. The necessity of the condition is a consequence of $\$ 4$, Theorem (a). Suppose conversely the validity of our condition. We infer from $(F)$ and the theorem of $\$ 5$ the existence of endomorphisms $\epsilon$ and $\kappa$ in $\theta$ with the following properties: $\eta \kappa=\kappa \eta=\epsilon, \kappa \epsilon=\epsilon \kappa=\kappa, L=R(\eta-\epsilon \eta)$. Hence we have in particular

$$
\epsilon=\eta \kappa=\eta \epsilon \kappa=\eta^{2} \kappa^{2}=\eta^{2} \epsilon \kappa^{2}=\eta^{3} \kappa^{3}=\cdots=\eta^{i} \kappa^{i}=\cdots
$$

so that $\epsilon$ belongs to the cross cut of the ideals $\eta^{i} \theta$. But this cross cut is 0 , showing that $\epsilon=0$. Hence $R(\eta)=R(\eta-\epsilon \eta)=L$, proving that $\eta$ is a nil endomorphism, as we claimed.

Remark. It is easy to construct examples showing that Theorem 2 would fail to be true without the hypothesis $(F)$.

THEOREM 3. If $\alpha$ and $\beta$ are endomorphisms in $\theta(A)$ such that $\alpha+\beta+\alpha \beta=0$, then $\alpha \beta=\beta \alpha, R(\alpha)=R(\beta)$, and $\alpha$ and $\beta$ have the same complements.

Proof. We precede the proof proper by the proofs of two propositions which do not involve any use of hypothesis $(F)$.

(1) $x \alpha^{i}=0$ for $0<i$ implies $x \beta^{i}=0$.

This contention we prove by complete induction with respect to $i$. First, if $x \alpha=0$, then $0=x(\alpha+\beta+\alpha \beta)=x \alpha+x \beta+x \alpha \beta=x \beta$, proving our contention for $i=1$. Second we assume the validity of our contention for some positive $i$. Suppose that $x$ is an element in $L$ such that $0=x \alpha^{i+1}$. Then $y=x \alpha$ is an element such that $y \alpha^{i}=0$; it follows from our induction hypothesis that $y \beta^{i}=0$. Hence

$$
\begin{aligned}
0 & =x(\alpha+\beta+\alpha \beta) \beta^{i}=x \alpha \beta^{i}+x \beta^{i+1}+x \alpha \beta^{i+1} \\
& =y \beta^{i}+x \beta^{i+1}+y \beta^{i} \beta=x \beta^{i+1},
\end{aligned}
$$

completing the inductive proof of (1).

From (1) we infer in particular that:

(1') $R(\alpha) \leqq R(\beta)$. 
(2) If $x \alpha^{i}=0$ for $0<i$, then $x \alpha \beta=x \beta \alpha$.

We prove this contention by complete induction with respect to $i$. First, if $x \alpha=0$, then we infer from (1) that $x \beta=0$ so that $x \alpha \beta=0=x \beta \alpha$, proving our contention for $i=1$. Second, we assume the validity of our contention for some positive $i$. Suppose that $x$ is an element in $L$ satisfying $x \alpha^{i+1}=0$. Then $y=x \alpha$ is an element in $L$ satisfying $y \alpha^{i}=0$; we infer from the induction hypothesis that $y \alpha \beta=y \beta \alpha$. Thus

$$
\begin{aligned}
0 & =x(\alpha+\beta+\alpha \beta) \alpha=x \alpha^{2}+x \beta \alpha+x \alpha \beta \alpha=x \alpha^{2}+x \beta \alpha+y \beta \alpha \\
& =x \alpha^{2}+x \beta \alpha+y \alpha \beta=x \alpha^{2}+x \beta \alpha+x \alpha^{2} \beta .
\end{aligned}
$$

But $0=x \alpha(\alpha+\beta+\alpha \beta)=x \alpha^{2}+x \alpha \beta+x \alpha^{2} \beta$; hence $x \alpha \beta=x \beta \alpha$, as we claimed.

From (2) we infer that:

(2') $x \alpha \beta=x \beta \alpha$ for $x$ in $R(\alpha)$.

By (F) there exist complements of $\alpha$. Denote by $C$ some complement of $\alpha$. If $x$ is an element in $C$, then $x \alpha$ is in $C=C \alpha$. Since $C \leqq A$ is abelian, an admissible endomorphism $\sigma$ of $C$ is defined by the rule $x \sigma=x+x \alpha$. If $x$ is an element in $C$ such that $x \sigma=0$, then

$$
x=x+x(\alpha+\beta+\alpha \beta)=x+x \alpha+(x+x \alpha) \beta=x \sigma+x \sigma \beta=0
$$

so that $\sigma$ is an admissible isomorphism of $C$ into itself. But $C$ is a complement and therefore a retract which is part of $A$. It follows from Theorem 1 that $C \sigma=C$. Consequently $\sigma$ is a proper admissible automorphism of $C$ and its inverse $\sigma^{-1}$ is well defined. If $x$ is an element in $C$, then $0=x \sigma^{-1}(\alpha+\beta+\alpha \beta)$ $=x \sigma^{-1} \alpha+x \beta$, proving that $x \beta$ too is in $C$. Consequently we may define an admissible endomorphism $\tau$ of $C$ by the equation $x \tau=x+x \beta$ for $x$ in $C$, and one verifies readily that $x \sigma \tau=x$ for every $x$ in $C$. Since $\sigma$ is a proper automorphism of $C$, it follows that $\sigma$ and $\tau$ are reciprocal automorphisms of $C$. Hence $x=x \tau \sigma=(x+x \beta)+(x+x \beta) \alpha$ for $x$ in $C$, or $x \alpha+x \beta+x \beta \alpha=0$ for $x$ in $C$; thus we have shown that $x \beta \alpha=x \alpha \beta$ for $x$ in $C$. Combine this result with (2') and $L=C+R(\alpha)$; it follows that $\alpha \beta=\beta \alpha$.

Consequently $\alpha+\beta+\beta \alpha=0$, and it follows from (1')-by symmetry-that $R(\beta) \leqq R(\alpha)$ and that consequently $R(\alpha)=R(\beta)$. We have already shown that $\beta$ induces an endomorphism in $C$ which is an isomorphism because of $R(\alpha)$ $=R(\beta)$. It follows now from Theorem 1 that $\beta$ induces a proper automorphism in $C$, showing that $C$ is a complement of $\beta$ too; now it is clear how to complete the proof.

Remark. It is a well known fact that Theorem 3 ceases to be valid without hypothesis $(\mathrm{F})$.

We are now ready to prove the main result of the present section.

THEOREM 4. The following properties of the endomorphism $\eta$ in $\theta(A)$ imply each other:

(a) $\eta$ belongs to the radical $\mathrm{P}$ of $\theta$. 
(b) The right ideal $\eta \theta$ does not contain an idempotent, not 0 .

(c) Every endomorphism in the right ideal $\eta \theta$ is a nil endomorphism.

(d) Every endomorphism in the right ideal generated by $\eta$ is a nil endomorphism.

Proof. If (a) is satisfied by $\eta$, then every element in the right ideal generated by $\eta$ is quasi regular. If $\epsilon$ is an idempotent in $\eta \theta$, then $\epsilon$ and $-\epsilon$ belong to the right ideal generated by $\eta$. Thus $-\epsilon$ possesses a quasi inverse $\epsilon^{\prime}$ satisfying $\epsilon \epsilon^{\prime}=\epsilon^{\prime} \epsilon$ and $-\epsilon+\epsilon^{\prime}-\epsilon \epsilon^{\prime}=0$. Premultiplying this equation by the idempotent $\epsilon$ we obtain $0=-\epsilon+\epsilon \epsilon^{\prime}-\epsilon \epsilon^{\prime}=-\epsilon$ or $\epsilon=0$, proving that (b) is a consequence of (a).

Suppose next the validity of (b) and assume that $\alpha$ is in $\eta \theta$. We infer from the hypothesis $(\mathrm{F})$ and from the theorem of $\$ 5$ the existence of endomorphisms $\epsilon$ and $\gamma$ in $\theta$ meeting the following requirements: $\kappa \gamma=\gamma \kappa=\epsilon, \gamma \epsilon=\epsilon \gamma$ $=\gamma, L=R(\kappa-\epsilon \kappa)$. It is clear that $\epsilon$ is in $\kappa \theta \leqq \eta \theta$. Furthermore $\epsilon^{2}=\kappa \gamma \epsilon=\kappa \gamma$ $=\epsilon$ is an idempotent; it follows from (b) that $\epsilon=0$. Consequently $L=R(\kappa)$, showing that $\kappa$ is a nil endomorphism. Thus (c) is a consequence of (b).

Assume next the validity of (c). If $\kappa$ is an endomorphism in the right ideal generated by $\eta$, then we infer from the hypothesis $(F)$ and the theorem of $\$ 5$ the existence of endomorphisms $\epsilon$ and $\gamma$ in $\theta$ with the following properties:

$$
\kappa \gamma=\gamma \kappa=\epsilon, \quad \gamma \epsilon=\epsilon \gamma=\gamma, \quad L=R(\kappa-\epsilon \kappa) .
$$

Since $\kappa$ is in the right ideal generated by $\eta$, there exists an integer $i$ and an element $\rho$ in $\theta$ such that $\kappa=i \eta+\eta \rho$. Hence $\epsilon=\kappa \gamma=\eta(i \gamma+\rho \gamma)$ is in $\eta \theta$; it follows from (c) that $L=R(\epsilon)$. But $\epsilon$ is an idempotent endomorphism of $L$, and hence $L=R(\epsilon)$ is the kernel of $\epsilon$ so that $\epsilon=0$. Consequently $L=R(\kappa)$ so that $\kappa$ is a nil endomorphism of $L$, showing that (d) is a consequence of (a).

It is finally a consequence of $\$ 4$, Corollary 2 , that (d) implies (a), completing the proof.

COROLlARY 1. The endomorphism $\eta$ in the ring $\theta(A)$ belongs to the radical $\mathrm{P}$ of $\theta$ if (and only if) $\eta \theta \leqq \mathrm{P}$.

Proof. If $\eta \theta \leqq \mathrm{P}$, and if $\epsilon$ is an idempotent in $\eta \theta$, then $\epsilon \theta \leqq \mathrm{P}$. But $\epsilon$ is in $\epsilon \theta$; and hence it follows from Theorem 4 that $\epsilon=0$. Since 0 is the only idempotent in $\eta \theta$, it follows from Theorem 4 that $\eta$ is in $\mathrm{P}$.

CoRollary 2. The right ideal $\Delta$ in the ring $\theta(A)$ is part of the radical $\mathbf{P}$ of $\theta$ if, and only if, 0 is the only idempotent in $\Delta$.

Proof. It is an immediate consequence of Theorem 4 that 0 is the only idempotent in the radical $P$. Thus $\Delta \leqq P$ implies that 0 is the only idempotent in $\Delta$. If conversely $\Delta$ is not part of $P$, then there exists an endomorphism $\eta$ in $\Delta$ which is not in $\mathrm{P}$; it follows from Theorem 4 that $\eta \theta$, and therefore $\Delta$, contains an idempotent different from 0 . 
Corollary 3. Suppose that $\epsilon$ is an idempotent in $\theta(A)$. Then there exists an idempotent $\rho \neq 0$ in $\theta$ which satisfies $\epsilon \rho=\rho \epsilon=0$ if, and only if, there exists an element $\tau$ in $\theta$, but not in the radical $\mathrm{P}$ of $\theta$, such that $\epsilon \tau=0$.

Proof. The necessity of our condition is a consequence of the fact that by Theorem 4 the radical does not contain idempotents not 0 . Assume conversely the existence of an element $\tau$ in $\theta$ which does not belong to $\mathbf{P}$ and satisfies $\epsilon \tau=0$. We infer from Theorem 4 the existence of an idempotent $\sigma \neq 0$ in $\tau \theta$. We have $\epsilon \sigma=0$, since $\epsilon \tau \theta=0$. Let $\rho=\sigma-\sigma \epsilon$. Then $\rho \neq 0$, since otherwise $\sigma=\sigma^{2}=(\sigma \epsilon) \sigma=0$. Furthermore $\rho^{2}=\sigma^{2}-\sigma^{2} \epsilon-\sigma \epsilon \sigma,+\sigma \epsilon \sigma \epsilon=\sigma-\sigma \epsilon=\rho$ is an idempotent. Finally $\rho \epsilon=\sigma \epsilon-\sigma \epsilon^{2}=0$ and $\epsilon \rho=\epsilon \sigma-\epsilon \sigma \epsilon=0$, showing that $\rho$ is the desired idempotent.

The preceding criterion may, sometimes, be used successfully to construct "maximal" idempotents. From Theorem 4 one may deduce, among other things, that minimal right (or left) ideals are annihilated by the radical.

Remark. Corollary 1 and the fact that Theorem 4 (a) implies Theorem 4 (b) hold for arbitrary rings; see Jacobson [2]. It has recently been pointed out by I. Kaplansky [1] that the implication of Theorem 4 (a) by Theorem 4 (d) holds also for complete topological rings with group neighborhoods of 0 .

7. Indecomposability. Suppose that $A$ is an abelian, admissible subgroup of the $M$-loop $L$. As before we assume that $A$ meets requirement 6, (F). Then we may define indecomposability of $A$ with respect to $L$ in a weak form (W) and in a strong form (S) as follows:

(S) 0 is the only admissible retract of the $M$-loop $L$ which is part of $A$.

(W) 0 is the only admissible retract of the $M$-loop $L$ which is part of $A$ and different from $\operatorname{L\theta }(A)$.

Assume that $L \theta \neq 0$, a hypothesis that we shall retain during the following discussion. Then it is clear that (S) implies (W). But (W) implies (S) if, and only if, $L \theta$ is not a retract of $L$; and it follows from Theorem 1 of $\$ 2$ that the latter property is equivalent to the absence of right identity elements in the ring $\theta$.

THEOREM 1. The following properties of $A$ and $L$ imply each other.

(i) $\theta=\mathbf{P}$.

(ii) Every endomorphism in $\theta$ is a nil endomorphism.

(iii) Property (S).

Proof. The equivalence of (i) and (ii) is an immediate consequence of $\$ 6$, Theorem 4 (a), (d). Property (S) is equivalent to the assertion that 0 is the only idempotent in the ring $\theta$; now the equivalence of (i) and (iii) is an immediate consequence of $\S 6$, Theorem 4 (a), (b).

For a convenient enunciation of the next theorem we need the following concept: If $\eta$ and $\kappa$ are elements in the ring $\theta$, such that $\eta \kappa$ is a right identity element of $\theta$, then $\kappa$ is a right-inverse of $\eta$. (See Baer [1] for this and related concepts.) 
TheOREM 2. Property (W) is satisfied by $A$ and $L$ if, and only if, every element in $\theta$ without a right inverse belongs to the radical $\mathrm{P}$.

Remark. This is a generalization of Jacobson [1, Theorem 3, p. 57].

Proof. It is a consequence of $\S 6$, Theorem 4 that elements in $\theta$ which possess a right inverse do not belong to the radical, since a right identity element is an idempotent, not 0 . Thus the condition of the present theorem may be restated as follows:

(I) The radical $\mathrm{P}$ of $\theta$ is the set of all the elements in $\theta$ without a right inverse.

It is a fairly immediate consequence of the proof of $\$ 2$, Theorem 1 that our condition (W) is equivalent to the following assertion:

$\left(\mathrm{W}^{\prime}\right)$ If $\epsilon$ is an idempotent, not 0 , in $\theta$, then $\epsilon$ is a right identity element of $\theta$.

From $\left(\mathrm{W}^{\prime}\right)$ and $\S 6$, Theorem 4 , one deduces that elements in $\theta$, but not in the radical $P$, possess right inverses, showing that (W) implies (I). If conversely $(\mathrm{I})$ is satisfied by $\theta$, and if $\epsilon$ is an idempotent, not 0 , in $\theta$, then we infer from $\S 6$, Theorem 4 , that $\epsilon$ is not in the radical $P$; and hence it follows from (I) that there exists an element $\rho$ in $\theta$ such that $\epsilon \rho$ is a right identity element of $\theta$. But then $\epsilon=\epsilon(\epsilon \rho)=\epsilon^{2} \rho=\epsilon \rho$ is a right identity element in $\theta$, proving that $\left(\mathrm{W}^{\prime}\right)$ and therefore $(\mathrm{W})$ is a consequence of $(\mathrm{I})$.

R. BAER

\section{BIBLIOGRAPHY}

1. Inverses and zero-divisors, Bull. Amer. Math. Soc. vol. 48 (1942) pp. 630-638.

2. Radical ideals, Amer. J. Math. vol. 65 (1943) pp. 537-568.

3. The homomorphism theorems for loops, Amer. J. of Math. vol. 67 (1945) pp. 450-460.

4. Splitting endomorphisms, Trans. Amer. Math. Soc. vol. 61 (1947) pp. 509-517.

H. Fitring

1. Die Theorie der Automorphismenringe abelscher Gruppen und ihr Analogon bei nichtkommutativen Gruppen, Math. Ann. vol. 107 (1933) pp. 514-543.

2. Über die direkten Produktzerlegungen einer Gruppe in direkt unzerlegbare Faktoren, Math. Zeit. vol. 39 (1934) pp. 16-30.

N. JACOBSON

1. The theory of rings, Mathematical Surveys, vol. 2, New York, 1943.

2. The radical and semi-simplicity for arbitrary rings, Amer. J. Math. vol. 67 (1945) pp. 300-320.

I. KAPLANSKY

1. Topological rings, Amer. J. Math. vol. 69 (1947) pp. 153-183.

V. KOŘ́́NEK.

1. Sur la décomposition d'un groupe en produit direct des sousgroupes, Casopis pro péstován! matematiky a fysiky vol. 66 (1937) pp. 261-286.

S. Perlis

1. A characterization of the radical of an algebra, Bull. Amer. Math. Soc. vol. 48 (1942) pp. 128-132.

UNIVERSITY OF ILLINOIS, URBANA, ILL. 